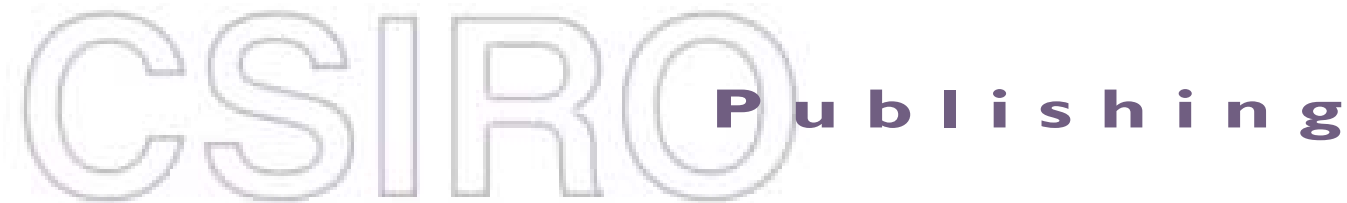

\section{Publications of the Astronomical Society of Australia}

Volume 19, 2002

(C) Astronomical Society of Australia 2002

An international journal of astronomy and astrophysics

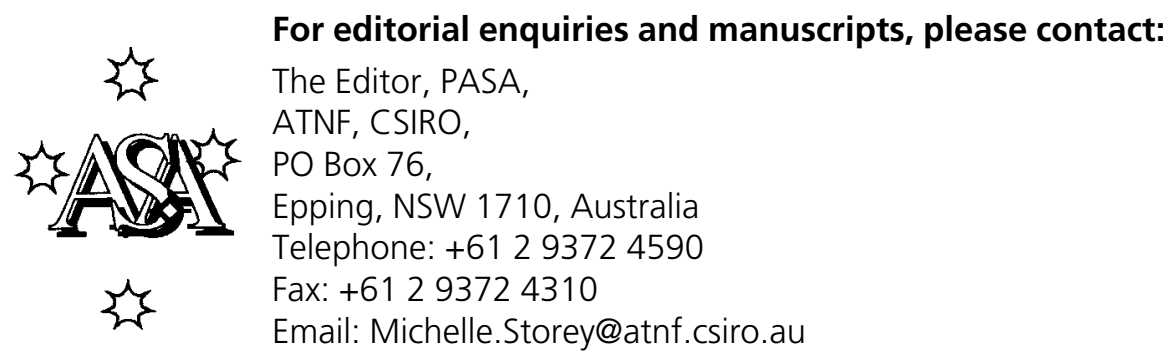

For general enquiries and subscriptions, please contact: CSIRO Publishing PO Box 1139 (150 Oxford St)

Collingwood, Vic. 3066, Australia

Telephone: +6139662 7666

Fax: +61 396627555

Email: publishing.pasa@csiro.au

C S I RO

PUBLISHING Published by CSIRO Publishing

for the Astronomical Society of Australia

www.publish.csiro.au/journals/pasa 


\title{
Big Bang Nucleosynthesis, Population III, and Stellar Genetics in the Galactic Halo*
}

\author{
Sean G. Ryan \\ Department of Physics and Astronomy, The Open University, Walton Hall, \\ Milton Keynes MK7 6AA, United Kingdom \\ s.g.ryan@open.ac.uk \\ Received 2001 September 10, accepted 2001 November 5
}

\begin{abstract}
The diverse isotopic and elemental signatures produced in different nucleosynthetic sites are passed on to successive generations of stars. By tracing these chemical signatures back through the stellar populations of the Galaxy, it is possible to unravel its nucleosynthetic history and even to study stars which are now extinct. This review considers recent applications of 'stellar genetics' to examine the earliest episodes of nucleosynthesis in the universe, in Population III stars and the Big Bang.
\end{abstract}

Keywords: stars: abundances - stars: Population II - Galaxy: abundances - Galaxy: formation Galaxy: halo

\section{Stellar Genetics}

Nucleosynthesis in the first few minutes following the Big Bang produced five isotopes in significant quantities: ${ }^{1} \mathrm{H},{ }^{2} \mathrm{H},{ }^{3} \mathrm{He},{ }^{4} \mathrm{He}$, and ${ }^{7} \mathrm{Li}$. The last of these accounts for less than one part per billion. All other elements were produced later, either in stars or as a consequence of stellar evolution.

Stellar nucleosynthesis theory predicts the yields of a wide range of isotopes, but in most astronomical spectra only elemental (rather than isotopic) abundances can be measured because the wavelength shift between different isotopes of an element is usually well below the intrinsic line width set by thermal and pressure broadening. Amongst the few exceptions are ${ }^{1} \mathrm{H}$ and ${ }^{2} \mathrm{H}$ (e.g. Linsky et al. 1995; Burles, Kirkman, \& Tytler 1999), and ${ }^{6} \mathrm{Li}$ and ${ }^{7} \mathrm{Li}$ (Smith, Lambert, \& Nissen 1993), whose isotope shifts are particularly large because of their small atomic masses. Isotope shifts are usually larger for molecular features, and ${ }^{12} \mathrm{C}$ and ${ }^{13} \mathrm{C}$ (e.g. Brown \& Wallerstein 1989) and certain $\mathrm{Mg}$ isotopes (e.g. Shetrone 1996) can be distinguished in $\mathrm{CH}$ and $\mathrm{MgH}$ bands. Although isotope shifts become even smaller for higher atomic masses, differences in the hyperfine structure of odd and even isotopes may allow constraints to be placed on isotope ratios for some elements as heavy as Ba (Magain 1995) and Eu (Hauge 1972). Nevertheless, stellar spectral measurements are generally limited to elemental rather than isotopic abundances.

By measuring the time-evolution of abundances coming from a variety of nucleosynthesis sites - see Figure 1 such as AGB stars, the cores of supernovae, and various radial zones in the envelopes of massive stars, it is possible to discover the roles of a wide range of stars over the history of the Galaxy. This is the activity I have branded

* Invited Talk, Annual Conference of the Astronomical Society of Australia, Lorne, 2-4 July 2001. 'stellar genetics': using the isotopic and elemental signatures that are passed from one stellar generation to the next to trace the particular nucleosynthesis reactions that have occurred in the past, and hence also to trace the sites of those reactions even when these may no longer exist. For example, low-metallicity asymptotic giant branch (AGB) stars have all now become white dwarfs, but it is nevertheless possible to study the internal structure of those AGB stars via the material they have dumped onto the surface of longer-lived companions (Ryan et al. 2001a).

The supernovae of massive stars are important early contributors to the enrichment of the Galaxy. Stars whose main sequence mass exceeds $8-10 \mathrm{M}_{\odot}$ are expected to become core collapse (type II) supernovae on timescales $<50 \mathrm{Myr}$, as short as $\sim 1 \mathrm{Myr}$ for stars of a few tens of solar masses. SN II produce of order ten times more oxygen than iron (by mass), whereas SN Ia, with lower-mass progenitors and hence longer time lags, produce of order seven times more iron than oxygen (e.g. Timmes, Woosley, $\&$ Weaver 1995). Consequently, the abundance ratios of oxygen and the $\alpha$-elements ${ }^{1}$ to iron are higher in Galactic halo stars than in the Sun (e.g. Tinsley 1979; Norris, Ryan, \& Beers 2001).

Although supernovae have been widely modelled, the role of stellar winds in removing processed material from highly luminous stars has not yet been widely investigated for the early enrichment of the halo, presumably because of uncertainties in the treatment of mass loss and its lower efficiency in metal-poor environments. However, the unexplained high levels of carbon enrichment in the early Galaxy, where perhaps $10 \%$ of stars have carbon-toiron ratios up to 100 times solar (Norris, Ryan, \& Beers 1997a; Barbuy et al. 1997; Rossi, Beers, \& Sneden 1999)

\footnotetext{
${ }^{1} \alpha$-elements are those relatively abundant light elements whose nuclei may be viewed as multiples of He nuclei, resulting either from He burning or further fusion of He-burning products.
} 


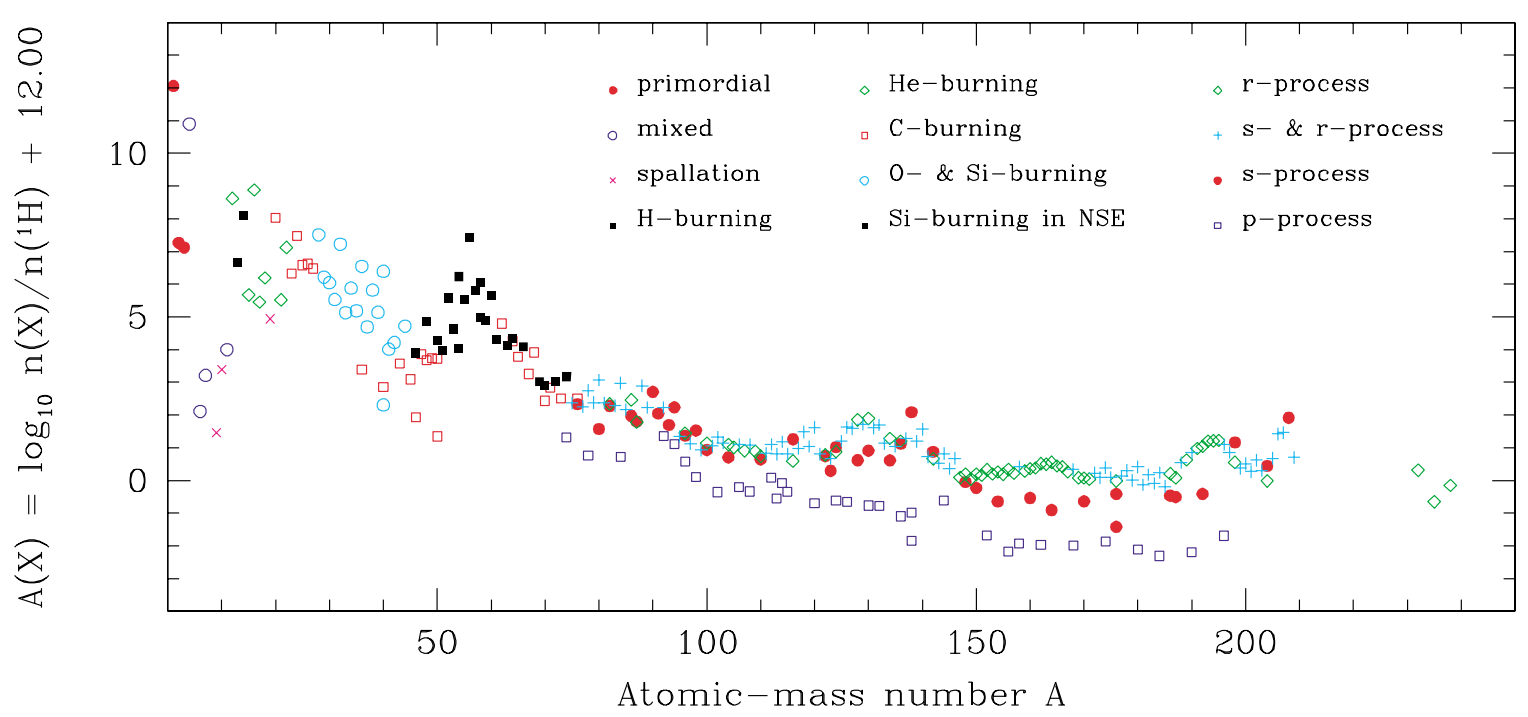

Figure 1 Isotopic abundances arranged by atomic mass number A. The legend indicates the main nucleosynthesis pathway for each species. (Based on data from Lang 1980, Table 38.)

suggest that more attention should be devoted to investigating this means of enriching the early interstellar medium. This is not to say that stellar winds are the only way of effecting large carbon excesses; indeed, the carbon-rich stars show a range of other signatures including s-process enhancements (Norris et al. 1997a), r-process enhancements (Cowan et al. 1995), and normal neutron-capture element abundances (Norris, Ryan, $\&$ Beers 1997c; Aoki et al. 2002). Nevertheless, one may speculate that mass loss may help produce high carbon-toiron ratios without producing neutron-capture elements if the ratio of black hole remnants to neutron star remnants is greater in metal-poor than metal-rich populations, as it might be since metal-poor stars are more compact and hence sit deeper in their gravitational potential wells. This might manifest itself in a lowering of the progenitor mass which corresponds to the division between (low-mass) neutron star remnants and (high-mass) black hole remnants in metal-poor populations. (This uncertain value is sometimes set in the range $30-50 \mathrm{M}_{\odot}$ in Galactic chemical evolution calculations; see also Prantzos (1994).) This would allow stellar winds to enrich the interstellar medium in carbon, yet avoid an associated enrichment in iron due to fall back onto a neutron star.

Stars below the mass limit for SN II become AGB stars which are responsible for most Galactic nucleosynthesis of carbon and s-process elements, but because of their lower masses they enrich the Galaxy after the first $\mathrm{SN}$ II. Models of $8-10 \mathrm{M}_{\odot}$ supernovae as the source of r-process nuclei suggest that these appeared as the Galaxy reached $[\mathrm{Fe} / \mathrm{H}] \simeq-3.0$ (e.g. Mathews \& Cowan 1990), which implies that AGB stars will enrich the halo only once this metallicity has been reached. This makes AGB stars unsuitable as the source of the carbon excess in lower metallicity stars. Also, the s-process contribution is insignificant until an even higher metallicity is reached, because of the need for pre-existing seed nuclei
(Spite \& Spite 1978; Truran 1981). The numerical model of Travaglio et al. (1999) suggests that the s-process appears only at $[\mathrm{Fe} / \mathrm{H}] \simeq-1$, though Pagel \& Tautvaišiené (1997) argue for its appearance as low as $[\mathrm{Fe} / \mathrm{H}]=-2.5$.

Although the science of stellar spectroscopy is more than a century old, many of the oldest stars in the Galactic halo have been discovered only in the last decade, as a result of the Beers, Preston, \& Shectman $(1985,1992)$ survey and its successors (e.g. Norris, Ryan, \& Beers 1998; Allende Prieto et al. 2000). Furthermore, detailed spectroscopic study of such stars requires échelle spectrographs on 4-10 m-class telescopes equipped with efficient electronic detectors. The remainder of this review highlights a number of issues concerning the very earliest stages of chemical evolution, namely Big Bang nucleosynthesis and the lack of identifiable Population III stars. Recent reviews of the evolution of other elements in the early phases of the Galaxy can be found in Ryan (2001) and Ryan et al. (2001b).

\section{The Elusive Population III}

The term 'Population III' means different things to different people. For some it refers to very metal-poor stars, whereupon the distinction between Population III and Population II becomes blurred. More usefully it can be applied to the first generation of stars that formed from material enriched by Big Bang nucleosynthesis alone, prior to stellar nucleosynthesis occurring. This is the definition that will be adopted here. The massive stars of this first stellar generation were probably responsible for the re-ionisation of the universe, possibly at redshifts around $z \simeq 10$ (Tumlinson \& Shull 2000), before the formation of Galactic halo stars at a redshift $z \simeq 5$ (e.g. Edmunds \& Phillipps 1997).

It might be supposed that searches for extremely metalpoor stars should encounter some having Population III 
composition, but none has been recognised. In fact, few would ever have existed. Recall that a single $25 \mathrm{M}_{\odot}$ supernova is sufficient to enrich a $10^{6} \mathrm{M}_{\odot}$ primordial gas cloud to $[\mathrm{Fe} / \mathrm{H}] \simeq-3.5$ (Ryan, Norris, \& Bessell 1991), demonstrating that very little stellar evolution and nucleosynthesis is required to significantly enrich the interstellar medium. Furthermore, such supernovae will appear on timescales of only a few million years. Population III stars will be a very minor constituent of the Galaxy.

A more instructive question to ask is whether there is a lower limit on the metallicity of Population II stars. The answer appears to be 'yes'. Despite the systematic surveys cited above, no star more metal-deficient than CD-38 245 (Bessell \& Norris 1984) has been discovered, though a few of comparable metallicity have been found. Although a simple closed-box model for galactic chemical evolution is a poor model for the Galactic disk, it does a surprisingly good job of fitting the Galactic halo (Hartwick 1976; Ryan \& Norris 1991a) and predicts a factor of ten fewer stars for each factor of ten lower metallicity. However, the good fit breaks down once $[\mathrm{Fe} / \mathrm{H}]$ falls to -4 , and a substantial deficit of stars becomes apparent. Based on current halo samples, the simple model suggests that 11 stars with $[\mathrm{Fe} / \mathrm{H}]<-4$ should have been detected, whereas at most two are known (Norris 1999). The Poisson probability of this occurring by chance is just $0.12 \%$, strongly suggesting the deficit is real.

A number of potential explanations for the absence of the more metal-poor stars can be proposed:

- The lowest metallicity (Population III?) stars probably formed before the Galaxy had collapsed to the size of the halo, and hence the missing stars may have a greater radial scale than the halo.

- The initial mass function (IMF) may have had a lower limit above $0.8 \mathrm{M}_{\odot}$, so no main sequence or red giant branch Population III stars remain after $\sim 15$ Gyr (e.g. Nakamura \& Umemura 1999).

- Since the pre-main sequence contraction time of $0.8 \mathrm{M}_{\odot}$ stars is $\sim 10^{7}$ yr whereas the first supernovae would have exploded after only $\sim 10^{6} \mathrm{yr}$, some contamination of low-mass Population III stars may have occurred. However, since low-mass stars will have already contracted to the base of their Hayashi tracks and undergone deuterium burning on a $\sim 10^{6} \mathrm{yr}$ timescale, it is less likely that contamination from a supernova would be very effective.

- Population III stars may have accreted sufficient material on passages through the Galactic disk that their thin outer convective zones are now contaminated by heavier elements (e.g. Yoshii 1981). However, such accretion could not hide Population III giants, whose greater convection would give them lower metallicities than dwarfs (which is not observed), and their heavy element abundance ratios would resemble those of the accreted material, but halo abundance ratios are different from that of disk material (Ryan \& Norris 1991b).
So, Population III stars are undetected, but that does not mean we cannot study them. Thanks to stellar genetics, the yields of the first Population III supernovae are observable as the post-primordial composition of Population II stars. Comparisons of the abundances of extremely metalpoor halo stars with the yields predicted for Population III supernovae (e.g. Woosley \& Weaver 1995) search for concordance in the abundance ratios, with the aim of revealing the mass, supernova energy ranges, and possibly eventually the rotation of Population III stars, thus identifying which types of stars have contributed to the early chemical enrichment of the Galaxy (e.g. Norris et al. 2001).

\section{Primordial Isotopes}

\section{$3.1{ }^{4} \mathrm{He}$}

Most Population II stars are not hot enough to excite He spectral lines. Those that are hot enough show the effects of diffusion which, in the absence of convective mixing, causes elements to stratify in the stellar atmosphere. To find the primordial ${ }^{4} \mathrm{He}$ composition, it is necessary to measure $\mathrm{H}$ II regions in galaxies that are less chemically enriched than the Milky Way. By measuring the strengths of emission lines of hydrogen, helium, and various metals (especially oxygen and nitrogen), it is possible to derive relative abundances (e.g. Pagel et al. 1992).

The evolution of the helium mass fraction $Y$ with metallicity mass fraction $Z$ is revealed in a graph of $Y$ vs $Z$, which gives a straight line graph of the form $Y=Y_{\mathrm{p}}+(\mathrm{d} Y / \mathrm{d} Z) Z$. The primordial helium abundance $Y_{\mathrm{p}}$ is simply the intercept.

Challenges in deriving the primordial helium mass fraction $Y_{\mathrm{p}}$ include:

- Helium production does not always accompany metal production at the same rate. That is, $\mathrm{d} Y / \mathrm{d} Z$ is not the same for all objects. Wolf-Rayet stars enrich H II regions more in He than the metals suggest, and bias $Y_{\mathrm{p}}$ upwards. Fortunately, Wolf-Rayet stars have a spectral signature which allows the most troublesome cases to be detected (e.g. Pagel et al. 1992), but some bias may remain at a low level.

- H II regions are visible only because the gas is excited by the UV thermal radiation from hot stars embedded in the clouds. The absorption lines in the stellar spectra literally undermine the emission line intensities, resulting in artificially weak He emission lines, from which low values of $Y_{\mathrm{p}}$ would be derived (e.g. Olive \& Skillman 2001).

- It is often assumed that only UV thermal radiation from the hot stars ionises and excites the nebula gas, but there may also be some excitation by collisions between gas atoms or by re-absorption of the emitted radiation. The effect of ionisation correction factors is unclear (Ballantyne, Ferland, \& Martin 2000; Viegas, Gruenwald, \& Steigman 2000).

An illustration of how these factors have influenced primordial He estimates is given in Figure 2. All values are 


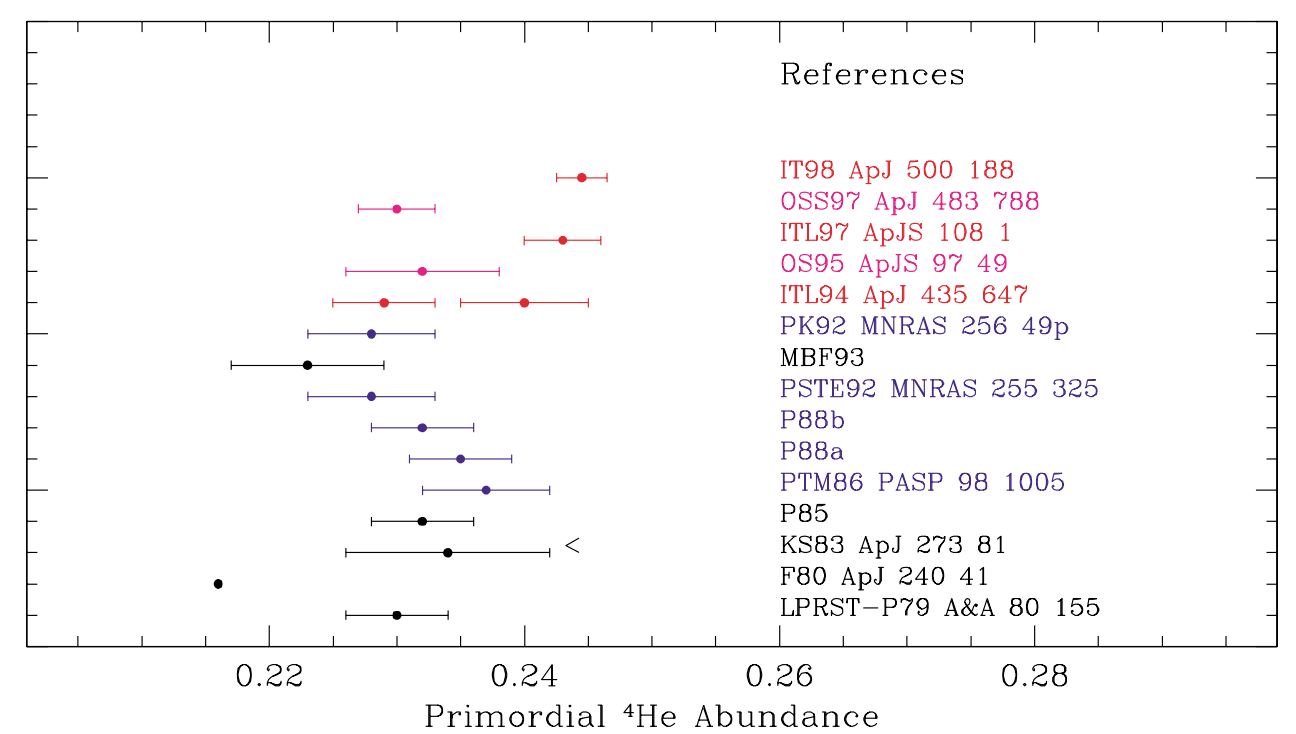

Figure 2 Measurements of the primordial helium abundance, 1979-1998. Horizontal points and bars give the inferred values, with references in the right-hand column.

close to $Y_{\mathrm{p}}=0.24$, but since the mid-1990s two streams of values have been obtained: a 'low' value near 0.230 , and a 'high' value near 0.245 . Moreover, the stated error bars are considerably smaller than this difference! The impact of these different values on the derived baryon density of the universe is discussed later.

\section{$3.2{ }^{3} \mathrm{He}$}

${ }^{3} \mathrm{He}$ is presently almost unusable as a diagnostic of Big Bang nucleosynthesis. In the solar system, direct measurements of ${ }^{3} \mathrm{He}$ atoms in the solar wind and meteorites can be made, but all of this material has passed through numerous stellar generations. The only other diagnostic is the $3.4 \mathrm{~cm}$ hyperfine transition of the ${ }^{3} \mathrm{He}$ II ion, analogous to the $21 \mathrm{~cm}$ hyperfine transition of ${ }^{1} \mathrm{H}$ I and the $92 \mathrm{~cm}$ hyperfine transition of ${ }^{2} \mathrm{H} \mathrm{I}$, which can be observed in $\mathrm{H}$ II regions and planetary nebulae.

To derive an abundance from the observations, an accurate model of the $\mathrm{H}$ II region or planetary nebula is needed. There is reason to doubt the suitability of current models, because different $\mathrm{H}$ II regions show a range of ${ }^{3} \mathrm{He}$ abundances that correlate with the mass of the $\mathrm{H}$ II region, suggesting that the ${ }^{3} \mathrm{He}$ has been depleted locally (Rood et al. 1995).

There are also large uncertainties in the interpretation of ${ }^{3} \mathrm{He}$ abundances. Stars are expected to both produce and destroy ${ }^{3} \mathrm{He}$ at different stages of their life. Until very recently, the expectation was that $1-2 \mathrm{M}_{\odot}$ stars would be net producers of ${ }^{3} \mathrm{He}$ while more massive stars would destroy it, but that the overall effect would be an increase in ${ }^{3} \mathrm{He}$ with metallicity. This was a problem, because it predicted much more ${ }^{3} \mathrm{He}$ than is observed. This may be resolved by recent developments in stellar evolution theory, through the proposal by Boothroyd \& Sackmann (1999) and Sackmann \& Boothroyd (1999) that mixing of material occurs deeper in stars than would be expected from standard convective theory, and that this leads to destruction of ${ }^{3} \mathrm{He}$ even in the lower mass stars previously believed to be net ${ }^{3} \mathrm{He}$ producers, making stars overall destroyers of this primordial isotope.

Given these significant problems, ${ }^{3} \mathrm{He}$ currently does not constrain primordial nucleosynthesis strongly. Bania, Rood, \& Balser (2001) describe their measurement ${ }^{3} \mathrm{He} / \mathrm{H}=(1.79 \pm 0.65) \times 10^{-5}$ as a 'reasonable approximation of the primordial value', while Bell (2000) reached the contrasting conclusion that 'the upper limit for the relative abundance of primordial ${ }^{3} \mathrm{He} \ldots$ is ${ }^{3} \mathrm{He}^{+} / \mathrm{H}^{+} \leq$ $2 \times 10^{-6}$.

\section{$3.3^{2} H$}

Deuterium is destroyed in stars, burning as $\mathrm{d}+\mathrm{p} \rightarrow{ }^{3} \mathrm{He}+$ $\gamma$. As there is no net source of deuterium in stars, its abundance has decreased steadily since the Big Bang, and any value measured today is a lower limit on the primordial value. Accurate measurements of the deuterium abundance can be made in solar system bodies and the interstellar medium of our Galaxy. The interstellar ' $\mathrm{D} \alpha$ ' line, which is displaced from the 'Lyman- $\alpha$ ' line of ${ }^{1} \mathrm{H}$ at $1216 \AA$ by $-0.33 \AA$, gives $\mathrm{D} / \mathrm{H}=1.6 \times 10^{-5}$, which is therefore a firm lower limit on the primordial value (Linsky et al. 1995). Models of the chemical evolution of the Galaxy can be applied to estimate how much deuterium has been destroyed over its lifetime, but this adds more uncertainty.

Much effort since the mid-1990s has gone into trying to measure deuterium in the interstellar medium of other galaxies, particularly in damped Lyman- $\alpha$ (DLA) absorption systems superimposed on quasar spectra at high redshift. The look-back times of objects at high redshifts offer the opportunity of looking directly into the past, and making measurements of the deuterium content 'back then'. Such values are expected to be closer to the 

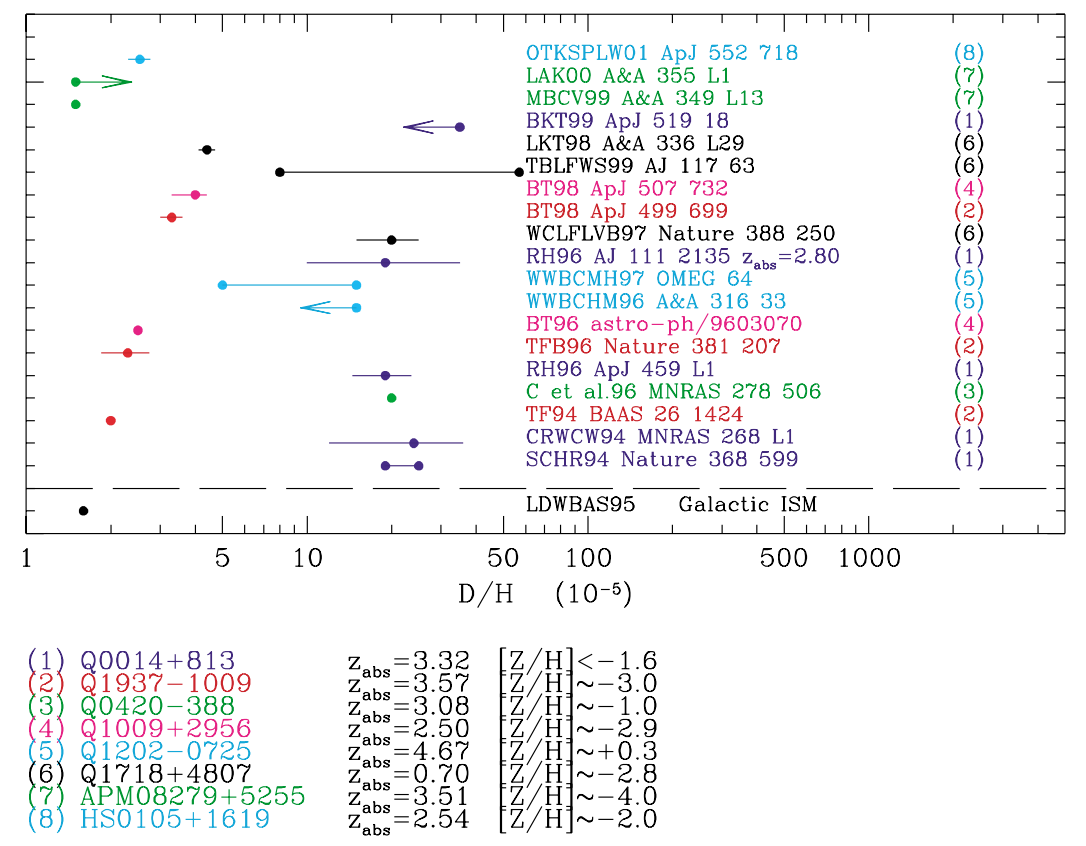

Figure 3 Measurements of the deuterium abundance in quasar absorption line systems, 1994-2001. (See Figure 2 for the layout.) The value measured in the interstellar medium (ISM) of the Milky Way is shown at the bottom for comparison. Below the figure are recorded the quasar names, the redshift of the measured absorption system (not the redshift of the quasar, $z_{\mathrm{em}}$ ), and the metallicity $\mathrm{Z}$ on a logarithmic scale relative to the Sun, where $[\mathrm{Z} / \mathrm{H}]=\log _{10}\left(\mathrm{Z} / \mathrm{Z}_{\text {Sun }}\right)$.

primordial one than values measured in the interstellar medium of our own Galaxy.

Because the abundance of deuterium is so much lower than that of hydrogen, large column densities of hydrogen are required before deuterium can be detected. Damped Lyman- $\alpha$ clouds have high column densities and are believed to be early galactic gas. Debate continues as to their nature: are they collapsed into flattened disks as in a mature galaxy like the Milky Way, or still distended like the halo of the Galaxy? The restriction to using high column density clouds is the source of several difficulties in obtaining reliable $\mathrm{D} / \mathrm{H}$ measurements at high redshift.

- Firstly, there is confusion over whether any single absorption line is due to deuterium or to another hydrogen line at a slightly lower redshift (e.g. Burles et al. 1999).

- Secondly, although it may be possible to measure the deuterium column density quite well, we also need to know the hydrogen column density if we are to obtain the ratio $\mathrm{D} / \mathrm{H}$. However, since the Lyman- $\alpha$ line is saturated (has absorbed all of the light), it is very difficult to measure its column density accurately. The H column density has to be obtained from other features in the spectrum.

Figure 3 shows measurements towards a range of quasars over 1994-2001. A considerable range of values has been obtained, including 'low' values around $\mathrm{D} / \mathrm{H}=(2-5) \times 10^{-5}$, and 'high' values around $\mathrm{D} / \mathrm{H}=$ $(15-30) \times 10^{-5}$.

The high and low values have very different implications. As any real measurement of deuterium sets a lower limit on the primordial abundance, if any of the 'high' measurements is correct, then the primordial value must be (at least!) an order of magnitude higher than that inferred from the Galactic ISM. However, the difficulty of knowing whether absorption just shortward of the damped Lyman$\alpha$ line is really due to deuterium weakens the arguments for very high $\mathrm{D} / \mathrm{H}$ ratios. 'Low' values have been measured in several systems, in particular the ones with low metallicity $[\mathrm{Z} / \mathrm{H}] \sim-3.0,-2.9$, and -2.8 . For the 'high' values to be correct, deuterium would have to be destroyed very quickly in the low metallicity systems despite their low metallicities indicating very little stellar processing. This seems unlikely (Tosi et al. 1998; Prantzos \& Ishimaru 2001). It is more likely that the lowest metallicity systems are more reliable measurements, and that the 'high' values are misleading due to the presence of additional ${ }^{1} \mathrm{H}$ absorption lines. Data are still being taken; it is perhaps a matter of opinion whether the issue has been settled, but it is probable that the 'low' deuterium measurements will win out over the 'high' ones once the dust settles. (See references to Figure 3.)

\section{$3.4{ }^{7} \mathrm{Li}$}

Lithium is measurable in the spectra of main sequence halo dwarfs. ${ }^{7} \mathrm{Li}$ is much less fragile than ${ }^{2} \mathrm{H}$, and as the outer layers of solar-mass metal-poor dwarfs do not mix to great depths, the surface Li in these stars is widely believed to be little changed since they formed $\sim 13 \mathrm{Gyr}$ ago. The main evidence in support of this is empirical: most solar-temperature, metal-poor dwarfs have almost the same Li abundance despite having different amounts of other elements and different masses (e.g. Spite \& Spite 1982). It is unlikely that all such stars could deplete or supplement their Big Bang Li complement and still end 
up with the same value after $\simeq 14$ Gyr. Theoretical models of stars differ in the amount of Li they preserve, and it is just conceivable that some mechanisms of $\mathrm{Li}$ destruction might conspire to deplete all stars by the same amount. However, the observations can tolerate little Li depletion even over the long lifetimes of these stars. For processing by rotationally-induced mixing, Ryan, Norris, \& Beers (1999) and Pinsonneault et al. (2001) infer limits $<0.1$ and $\simeq 0.2$ dex respectively. The difference in these values depends on (A) the frequency of the few stars with $\mathrm{Li}$ abundances significantly lower than the rest, (B) the size distribution of their deficiencies, and (C) whether the deviations are in fact due to the proposed mechanism (rotationally-induced mixing) and not some other. Small amounts of Li production may also be present in the stars that formed more recently from nucleosynthetically enriched material. This can be tracked analogously to the way He production is tracked by $\mathrm{d} Y / \mathrm{d} Z$. The main uncertainty remaining for $\mathrm{Li}$ is how accurately we know the temperatures of the stellar atmospheres, and therefore how well we can compute the Li abundances from their spectra. The estimate of the primordial $\mathrm{Li}$ abundance by Ryan et al. (2000) is $n(\mathrm{Li}) / n(\mathrm{H})=1.23_{-0.32}^{+0.68} \times 10^{-10}$.

Note, however, that even though the primordial $\mathrm{Li}$ abundance may be reasonably well known, there are several significant deficiencies in our understanding of $\mathrm{Li}$ processing in Population II and Population I stars. About $7 \%$ of Population II stars which would be expected to have normal Li abundances show extensive (essentially complete) destruction of the element. Investigations of other elements in these stars (Norris et al. 1997b; Ryan, Norris, \& Beers 1998) show heterogeneous behaviour and indicate that no single characteristic besides Li deficiency unites these stars. Ryan et al. (2001c) have suggested they may be blue-stragglers-to-be, sub-turnoff-mass products of classical blue-straggler formation mechanisms. There is also an unexplained contrast between the highly uniform $\mathrm{Li}$ abundances measured in halo field stars (Ryan et al. 1999) and the large spread of measurements for subgiants in the halo globular cluster M92 (Boesgaard et al. 1998).

\subsection{Putting It All Together}

We now put the observational constraints together and examine the concordance with theory. The standard Big Bang model assumes a uniform density and predicts the yields of the primordial isotopes for different values of that density. ${ }^{2}$ The predicted yield of ${ }^{4} \mathrm{He}$ is close to $24 \%$ for a wide range of densities, and the fact that this is so close to the real universe is a remarkable accomplishment of the model. It is even more remarkable that for ${ }^{2} \mathrm{H}$ and ${ }^{7} \mathrm{Li}$ the abundances are also in accord. For three isotopes spanning a range of 1 billion in abundance, the model is broadly in accord with observations.

\footnotetext{
${ }^{2}$ The density may be expressed variously as the baryon-to-photon ratio, $\eta$, or the baryon density as a fraction of the critical density, $\Omega_{B}$, where $\Omega_{B}=0.014 \times\left(\eta / 10^{-10}\right) \times\left(50 \mathrm{~km} \mathrm{~s}^{-1} \mathrm{Mpc}^{-1} / H_{0}\right)^{2}$.
}

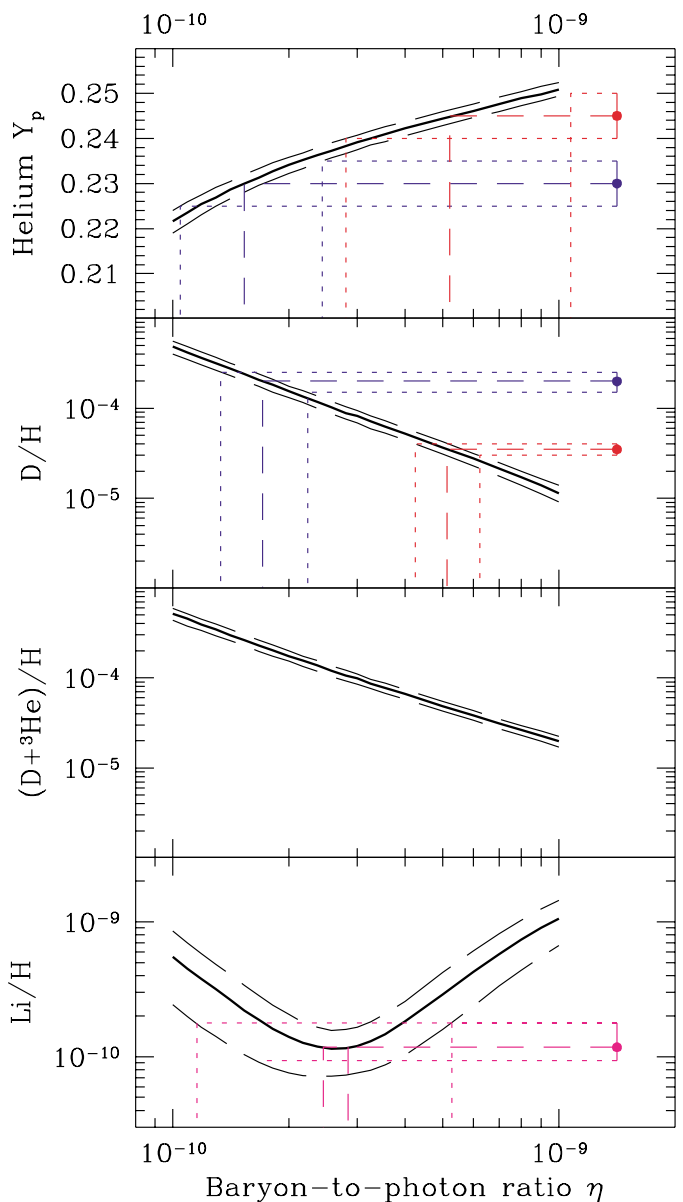

Figure 4 Solid curves: Calculations of yields of Big Bang nucleosynthesis for ${ }^{4} \mathrm{He},{ }^{2} \mathrm{H}$, the sum of ${ }^{2} \mathrm{H}$ and ${ }^{3} \mathrm{He}$, and ${ }^{7} \mathrm{Li}$, for different assumed baryon-to-photon ratios. Long dash curves: $95 \%$ confidence range for the nucleosynthesis calculations, primarily reflecting uncertainties in the nuclear reaction rates. Data symbols: current observational values, split for ${ }^{4} \mathrm{He}$ and ${ }^{2} \mathrm{D}$ into 'high' and 'low' values to reflect current systematic uncertainties.

Figure 4 presents the situation in more detail, and illustrates the different values of the derived ${ }^{4} \mathrm{He}$ and ${ }^{2} \mathrm{H}$ abundances, and the ${ }^{7} \mathrm{Li}$ value. No ${ }^{3} \mathrm{He}$ observations are shown. The predicted yield of $\mathrm{Li}$ is not monotonic with density, as a second nucleosynthesis pathway becomes effective for $\eta>2 \times 10^{-10}$. The well-defined Li abundance is therefore consistent with a wide range of density values. Two observational results are given for each of ${ }^{4} \mathrm{He}$ and ${ }^{2} \mathrm{H}$, the 'high' and 'low' values discussed above. Corrections for systematic errors in the ${ }^{4} \mathrm{He}$ analysis favour 'high' values, and the study of more damped Lyman- $\alpha$ systems favour the 'lower' range for $\mathrm{D}$, giving a value for $\eta \simeq 5 \times 10^{-10}$. (The slightly lower $95 \%$ confidence interval given by Ryan et al. (2000), $\eta=(1.7-3.9) \times 10^{-10}$, was based on a primordial ${ }^{4} \mathrm{He}$ abundance intermediate between the two observational values shown here.)

An independent measurement of the universal baryon density is possible from the power spectrum of the cosmic microwave background radiation (CMBR). The higherorder fluctuations above the main $\left(\simeq 1^{\circ}\right)$ peak are influenced by the propagation of sound waves, which reflects 
the baryon density. Measurements published in 2000 giving $\eta=10 \times 10^{-10}$ raised the spectre of incompatible Big Bang nucleosynthesis (BBN) and CMBR measurements of the baryon density (Hogan 2000). Fortunately, the earlier values have been superseded with the addition of more data such that the CMBR measurements now suggest a $95 \%$ confidence interval $\eta=(3.4-7.9) \times 10^{-10}$, which is certainly compatible with the BBN values cited above (Netterfield et al. 2001).

For an assumed Hubble constant of $63 \mathrm{~km} \mathrm{~s}^{-1} \mathrm{Mpc}^{-1}$, the value $\eta=5 \times 10^{-10}$ corresponds to $\Omega_{B}=0.043$. Not only is this well below the closure density, it is also a factor of ten below the matter density $\Omega_{M} \simeq 0.35$ inferred from other cosmological studies (Efstathiou et al. 1999), pointing to the continuing need for non-baryonic dark matter.

\section{Acknowledgments}

It is a pleasure to thank the organising committee of the 2001 Conference of the Astronomical Society of Australia for their support of my attendance at the meeting. The suggestions of an anonymous referee were gratefully received.

\section{References}

Allende Prieto, C., et al. 2000, AJ, 120, 1516

Aoki, W., Norris, J. E., Ryan, S. G., Beers, T. C., \& Ando, H. 2002, ApJ, 567, 1166

Ballantyne, D. R., Ferland, G. J., \& Martin, P. G. 2000, ApJ, 536, 773

Bania, T. M., Rood, R. T., \& Balser, D. S. 2001, BAAS, 198, 4106

Barbuy, B., Cayrel, R., Spite, M., Beers, T. C., Spite, F., Nordstrom, B., \& Nissen, P. E. 1997, A\&A, 317, L63

Beers, T. C., Preston, G. W., \& Shectman, S. A. 1985, AJ, 90, 2089

Beers, T. C., Preston, G. W., \& Shectman, S. A. 1992, AJ, 103, 1987

Bell, M. B. 2000, ApJ, 531, 820

Bessell, M. S., \& Norris, J. E. 1984, ApJ, 285, 622

Boesgaard, A. M., Deliyannis, C. P., Stephens, A., \& King, J. R. 1998, ApJ, 493, 206

Boothroyd, A. I., \& Sackmann, I.-J. 1999, ApJ, 510, 232

Brown, J. A., \& Wallerstein, G. 1989, AJ, 98, 1643

Burles, S., Kirkman, D., \& Tytler, D. 1999, ApJ, 519, 18

Burles, S., \& Tytler, D. 1996, astro-ph/9603070

Burles, S., \& Tytler, D. 1998, ApJ, 499, 699

Burles, S., \& Tytler, D. 1998, ApJ, 507, 732

Carswell, R. F., Rauch, M., Weymann, R. J., Cooke, A. J., \& Webb, J. K. 1994, MNRAS, 268, L1

Carswell, R. F., et al. 1996, MNRAS, 278, 506

Cowan, J. J., Burris, D. L., Sneden, C., McWilliam, A., \& Preston, G. W. 1995, ApJ, 439, L51

Edmunds, M. G., \& Phillipps, S. 1997, MNRAS, 292, 733

Efstathiou, G., Bridle, S. L., Lasenby, A. N., Hobson, M. P., \& Ellis, R. S. 1999, MNRAS, 303, L47

French, H. B. 1980, ApJ, 240, 41

Hartwick, F. D. A. 1976, ApJ, 209, 418

Hauge, O. 1972, SoPh, 27, 286

Hogan, C. 2000, Nature, 408, 47

Izotov, Y. I., \& Thuan, T. X. 1998, ApJ, 500, 188

Izotov, Y. I., Thuan, T. X., \& Lipovetsky, V. A. 1994, ApJ, 435, 647

Izotov, Y. I., Thuan, T. X., \& Lipovetsky, V. A. 1997, ApJS, 108, 1

Kunth, D., \& Sargent, W. L. W. 1983, ApJ, 273, 81

Lang, K. R. 1980, Astrophysical Formulae (Berlin: Springer-Verlag)
Lequeux, J., Rayo, J. F., Serrano, A., Peimbert, M., \& TorresPeimbert, S. 1979, A\&A, 80, 155

Levshakov, S. A., Agafonova, I. I., \& Kegel, W. H. 2000, A\&A, 355, L1

Levshakov, S. A., Kegel, W. H., \& Takahara, F. 1998, A\&A, 336, L29

Linsky, J. L., Diplas, A., Wood, B. E., Brown, A., Ayres, T. R., \& Savage, B. D. 1995, ApJ, 451, 335

Magain, P. 1995, A\&A, 297, 686

Mathews, G. J., Boyd, R. N., \& Fuller, G. M. 1993, ApJ, 403, 65

Mathews, G. J., \& Cowan, J. J. 1990, Nature, 345, 491

Molaro, P., Bonifacio, P., Centurion, M., \& Vladilo, G. 1999, A\&A, 349, L13

Nakamura, F., \& Umemura, M. 1999, ApJ, 515, 239

Netterfield, C. B., et al. 2001, astro-ph/0104460

Norris, J. E. 1999, in The Third Stromlo Symposium: The Galactic Halo, ASP Conf. Ser. 165, eds B. K. Gibson, T. S. Axelrod, \& M. E. Putman (San Francisco: ASP), 213

Norris, J. E., Ryan, S. G., \& Beers, T. C. 1997a, ApJ, 488, 350

Norris, J. E., Ryan, S. G., \& Beers, T. C. 1997c, ApJ, 489, L169

Norris, J. E., Ryan, S. G., \& Beers, T. C. 1998, ApJS, 123, 639

Norris, J. E., Ryan, S. G., \& Beers, T. C. 2001, ApJ, 561, 1034

Norris, J. E., Ryan, S. G., Beers, T. C., \& Deliyannis, C. P. 1997b, ApJ, 485, 370

Olive, K. A., \& Skillman, E. D. 2001, NewA, 6, 119

Olive, K. A., \& Steigman, G. 1995, ApJS, 97, 49

Olive, K. A., Steigman, G., \& Skillman, E. D. 1997, ApJ, 483, 788

O’Meara, J. M., Tytler, D., Kirkman, D., Suzuki, N., Prochaska, J. X., Lubin, D., \& Wolfe, A. M. 2001, ApJ, 552, 718

Pagel, B. E. J. 1988a, in Astro-Particle Physics, eds A. de Rujula, D. Nanapoulos, \& P. A. Shaver (Singapore: World Scientific), 399

Pagel, B. E. J. 1988b, in Evolutionary Phenomena in Galaxies, eds J. Beckman, \& B. E. J. Pagel (Cambridge: Cambridge University Press), 201

Pagel, B. E. J., \& Kazauskas, A. 1992, MNRAS, 256, 49p

Pagel, B. E. J., Simonson, E. A., Terlevich, R. J., \& Edmunds, M. G. 1992, MNRAS, 255, 325

Pagel, B. E. J., \& Tautvaišienè, G. 1997, MNRAS, 288, 108

Pagel, B. E. J., Terlevich, R. J., \& Melnick, J. 1986, PASP, 98, 1005

Peimbert, M. 1985, in Star Forming Dwarf Galaxies, eds D. Kunth, T. X. Thuan, \& J. T. T. Van (Paris: Ed. Forntières), 403

Pinsonneault, M. H., Walker, T. P., Steigman, G., \& Narayanan, V. K. 2001, astro-ph/0105439

Prantzos, N. 1994, A\&A, 284, 477

Prantzos, N., \& Ishimaru, Y. 2001, A\&A, 376, 751

Rood, R. T., Bania, T. M., Wilson, T. L., \& Balser, D. S. 1995, in The Light Element Abundances, ed. P. Crane (Berlin: Springer), 201

Rossi, S., Beers, T. C., \& Sneden, C. 1999, in The Third Stromlo Symposium: The Galactic Halo, ASP Conf. Ser. 165, eds B. K. Gibson, T. S. Axelrod, \& M. E. Putman (San Francisco: ASP), 264

Rugers, M., \& Hogan, C. J. 1996a, ApJ, 459, L1

Rugers, M., \& Hogan, C. J. 1996b, AJ, 111, 2135

Ryan, S. G. 2001, The Influence of Binaries on Stellar Population Studies, ed. D. Vanbeveren (Dordrecht: Kluwer), 491

Ryan, S. G., Aoki, W., Blake, L. A. J., Norris, J. E., Beers, T. C., Gallino, R., Busso, M., \& Ando, H. 2001b, MmSAI, in press

Ryan, S. G., Aoki, W., Norris, J. E., Beers, T. C., Gallino, R., Busso, M., \& Ando, H. 2001a, NuPhA, 688, 209c

Ryan, S. G., Beers, T. C., Kajino, T., \& Rosolankova, K. 2001c, ApJ, 547,231

Ryan, S. G., Beers, T. C., Olive, K. A., Fields, B. D., \& Norris, J. E. 2000, ApJ, 530, L57

Ryan, S. G., \& Norris, J. E. 1991a, AJ, 101, 1865

Ryan, S. G., \& Norris, J. E. 1991b, AJ, 101, 1835

Ryan, S. G., Norris, J. E., \& Beers, T. C. 1998, ApJ, 506, 892

Ryan, S. G., Norris, J. E., \& Beers, T. C. 1999, ApJ, 523, 654

Ryan, S. G., Norris, J. E., \& Bessell, M. S. 1991, AJ, 102, 303

Sackmann, I.-J., \& Boothroyd, A. I. 1999, ApJ, 510, 217 
Shetrone, M. D. 1996, AJ, 112, 2639

Smith, V. V., Lambert, D. L., \& Nissen, P.-E. 1993, ApJ, 408, 262

Songaila, A., Cowie, L. L., Hogan, C. J., \& Rugers, M. 1994, Nature, 368,599

Spite, F., \& Spite, M. 1982, A\&A, 115, 357

Spite, M., \& Spite, F. 1978, A\&A, 67, 23

Timmes, F. X., Woosley, S. E., \& Weaver, T. A. 1995, ApJS, 98, 617

Tinsley, B. M. 1979, ApJ, 229, 1046

Tosi, M., Steigman, G., Matteucci, F., \& Chiappini, C. 1998, ApJ, 498, 226

Travaglio, C., Galli, D., Gallino, R., Busso, M., Ferrini, F., \& Straniero, O. 1999, ApJ, 521, 691

Truran, J. W. 1981, A\&A, 97, 391

Tumlinson, J., \& Shull, J. M. 2000, ApJ, 528, L65

Tytler, D., Burles, S., Lu, L., Fan, X.-M., Wolfe, A., \& Savage, B. D. 1999, AJ, 117, 63
Tytler, D., \& Fan, X.-M. 1994, BAAS, 26, 1424

Tytler, D., Fan, X.-M., \& Burles, S. 1996, Nature, 381, 207

Viegas, S. M., Gruenwald, R., \& Steigman, G. 2000, ApJ, 531, 813 Wampler, E. J., Williger, G. M., Baldwin, J. A., Carswell, R. F., Hazard, C., \& McMahon, R. G. 1996, A\&A, 316, 33

Wampler, E. J., Williger, G. M., Baldwin, J. A., Carswell, R. F., McMahon, R. G., \& Hazard, C. 1997, in Origin of Matter and Evolution of Galaxies, eds T. Kajino, S. Kubono, \& Y. Yoshii (Singapore: World Scientific), 64

Webb, J. K., Carswell, R. F., Lanzetta, K. M., Ferlet, R., Lemoine, M., Vidal-Madjar, A., \& Bowen, D. V. 1997, Nature, 388, 250

Woosley, S. E., \& Weaver, T. A. 1995, ApJS, 101, 181

Yoshii, Y. 1981, A\&A, 97, 280 